\title{
AVALIAÇÃO DA CORROSÃO DAS LIGAS Ti-35Nb-xTa EM SOLUÇÃO DE SBF ANTES E APÓS ANODIZAÇÃO*
}

\author{
Thamyres Moura Limar ${ }^{1}$ \\ Gusttavo Reis Leite Matos $^{2}$ \\ Michelle Cardinale Souza Silva Macedo ${ }^{3}$ \\ Sandra Andreia Stwart de Araujo Souza ${ }^{4}$ \\ Reinan Tiago Fernandes ${ }^{5}$
}

\section{Resumo}

O presente trabalho, traz a analise eletroquímica, curvas de polarização, das ligas Ti$35 \mathrm{Nb}-\mathrm{xTa}(\mathrm{x}=0 ; 2,5 ; 5,0 ; 7,5) \mathrm{Ta}$ (\% em peso) anodizadas, a um potencial de $20 \mathrm{~V}$ e 1 hora e sem anodizar, para verificar o aumento da resistência a corrosão dessas ligas. O meio ao qual os ensaios foram realizados foi o sfb ( fluido corpóreo simulado).Obtendo como resultado que as ligas anodizadas apresentam maior resistênciaa corrosão nesse meio. A liga sem adição de tântalo obteve menor densidade de corrente, assim tendo uma maior resistência a corrosão após anodizada o que não ocorre sem a anodização.

Palavras-chave: Anodização; Ligas; Corrosão.

\section{CORROSION EVALUATION ALLOY TI-35NB-XTA IN SBF SOLUTION BEFORE AND AFTER ANODIZATION}

\section{Abstract}

The present work presents the electrochemical analysis, polarization curves of the Ti$35 \mathrm{Nb}-\mathrm{xTa}(\mathrm{x}=0 ; 2,5 ; 5,0 ; 7,5) \mathrm{Ta}(\mathrm{wt} \%)$ anodized alloys at a potential of $20 \mathrm{~V}$ And 1 hour and without anodizing, to verify the increase in the corrosion resistance of these alloys. The medium to which the tests were performed was the sfb (simulated bodily fluid). As a result, the anodized alloys present greater corrosion resistance in this medium. The alloy without addition of tantalum obtained a lower current density, thus having a higher resistance to corrosion after anodizing than does not occur without anodization.

Keywords: Anodizing; Alloys; Corrosion.

1 Ciencia e engenharia de materiais, mestrando,Estudante,Universidade Federal de Sergipe, São Cristóvão, SE, Brasil

2 Engenharia de Materiais, Graduação, Estudante, Universidade Federal de Sergipe, São Cristóvão, SE, Brasil

3 Engenharia Metalúrgica e de Materiais, Doutora, Professora, Departamento de Ciência e Engenharia de Materiais, Universidade Federal de Sergipe, São Cristóvão, SE, Brasil.

4 Engenharia Mecânica, Doutora, Professora, Departamento de Ciência e Engenharia de Materiais, Universidade Federal de Sergipe, São Cristóvão, SE, Brasil..

5 Ciencia e engenharia de materiais,mestrando,Estudante,Universidade Federal de Sergipe, São Cristóvão, SE, Brasil. 


\section{INTRODUÇÃO}

Diferentes biomateriais são usados e pesquisados para serem destinados a implantes, dentre eles polímeros, cerâmicas, metais e compósitos. Os implantes metálicos, devido suas propriedades mecânicas, são considerados a melhor opção para a substituição de longo prazo, especialmente em aplicações de suporte de carga, tais como as articulações de joelho, de quadril e dentárias[1 -2]

O titânio e suas ligas são os materiais metálicos mais comumente utilizados na fabricação de próteses e componentes ortopédicos, devido às suas propriedades mecânicas, elevada resistência à corrosão e biocompatibilidade [3].

Ao considerar o desempenho de um implante aplicado na reparação de tecidos duros, além das características e propriedades intrínsecas do material, como as propriedades mecânicas, um aspecto de suma importância é a superfície do mesmo, que tem relação íntima com sua ósseo-integração, recuperação e adaptação do tecido e potencial de corrosão [4].

Resultados da literatura sugerem que a chance de sucesso de ósseo-integração de implantes e a resistência a corrosão pode vir a aumentar com a camada de passivação de $\mathrm{TiO}_{2}$ espontânea, quando em contato com a atmosfera. Quando essa camada de $\mathrm{TiO}_{2}$ é nanoestruturada, a qual pode ser formada através de técnicas como a anodização, pesquisas comprovam um aumento dessas propriedades. Vale ainda ressaltar que as microestruturas das ligas têm um papel importante no controle de sua nanotopografia e resistência a corrosão, nesse caso, a fase $\beta$ é atrativa por facilitar a nucleação e crescimento da camada de oxido com uma estrutura mais ordenada[4 - 5].

Este trabalho propõe o estudo da resistência a corrosão da liga ternaria de titânio com os elementos $\beta$ estabilizantes Nióbio e tântalo, anodizadas, para modificações da superfície, onde tem por objetivo criar uma camada de oxido a ser estudada através de ensaios eletroquímicos.

\section{MATERIAIS E MÉTODOS}

As ligas a serem estudadas nesse trabalho, foram produzidas a partir de $\mathrm{Ti}, \mathrm{Nb}$ e $\mathrm{Ta}$ comercialmente puros. Os dois primeiros metais foram adquiridos em barras e placas que foram cortadas em pedaços menores e decapados, enquanto que 0 último foi recebido em pequenos pedaços isentos de óxidos.

A fusão foi realizada em um forno a arco com eletrodo de tungstênio não consumível em um cadinho de cobre refrigerado sob atmosfera controlada de argônio. Suas composições nominais são Ti-35Nb-(0;2,5; 5,0; 7,5\%)Ta (\% em peso). As ligas foram submetidos a tratamento térmico de homogeneização à temperatura de $1000^{\circ} \mathrm{C}$ por 12 horas sob atmosfera inerte. As amostras foram resfriadas ao forno até a temperatura ambiente com o intuito de eliminar heterogeneidades resultantes do processo de solidificação.

Em todas as amostras um fio de cobre foi soldado para estabelecer o contato elétrico e posteriormente as mesmas foram embutidas em resina poliéster de cura rápida. Em seguida, as superfícies das amostras foram lixadas até a lixa de grana 4000, limpas e desengorduradas em uma solução de água/acetona 1:1 em banho de ultrassom durante $15 \mathrm{~min}$.

As amostras a serem anodizadas foram levadas para a célula eletroquímica e conectadas a uma fonte de alimentação (anodo) e uma rede de platina (catodo), em um eletrólito contendo glicerol e NH4F 0,25\%. Foi aplicada uma tensão à célula, 
elevada gradativamente, $1 \mathrm{~V} / \mathrm{min}$ até atingir o valor final de $20 \mathrm{~V}$, que foi mantido constante por $1 \mathrm{~h}$ a temperatura ambiente. Após esse procedimento, os ensaios eletroquímicos foram realizados.

As curvas de polarização anódicas e as impedâncias foram realizadas usando uma célula eletroquímica convencional de três eletrodos, onde as ligas são os eletrodos de trabalho, o eletrodo de calomelano saturado foi usado como eletrodo de referência e uma haste de platina como contra eletrodo. O equipamento utilizado para as medidas foi um potenciostato/ galvanostato Aultolab 302N.. As curvas de polarização anódica foram obtidas partindo do PAC até $2 \mathrm{~V}$ de sobretensão, com uma velocidade de varredura de $5 \mathrm{mV} / \mathrm{s}$. O eletrólito usado foi uma solução de SBF (fluido corpóreo simulado).

\section{RESULTADOS E DISCUSSÃO}

\subsection{Ensaios Eletroquímicos}

A Figura 1 e a figura 2 mostram as curvas de polarização das ligas sem anodizar e anodizadas respectivamente. Todas as amostras estudadas apresentaram, neste meio, um comportamento típico de material que se passiva, onde tem-se uma região de passivação, caracterizada por um patamar de corrente, uma região onde ocorre a quebra do filme de óxido pelo surgimento de pites, caracterizado pelo aumento da corrente e finalmente um novo patamar de corrente é observado como conseqüência da repassivação das ligas. Esse comportamento de rapassivação tem sido observado por outros autores em ligas de titânio e segundo os mesmos essa capacidade que o material tem de se repassivar exibe um importante papel na resistência à corrosão da liga [6-7].

$\mathrm{Na}$ figura 1 observa-se que a liga sem tântalo, Ti-35Nb, apresenta densidades de correntes maiores, assim quando o Tântalo e adicionado tem se a redução das correntes, comportamento que não se repete quando as amostras são anodizadas. Uma possível possibilidade para que isso ocorra, e que o oxido formado sofra uma influencia do tântalo. A anodização se mostra eficiente pois quando comparadas as densidades de corrente das amostras anodizadas e sem anodizar, tem se densidade de correntes bem menores,o que indica maior ressitencia a corrosão,quase 10 vezes. Como já previsto na literatura, a técnica de anodização apresenta uma camada de oxido com uma resistência a corrosão maior do que a da liga sem sofrer o processo.

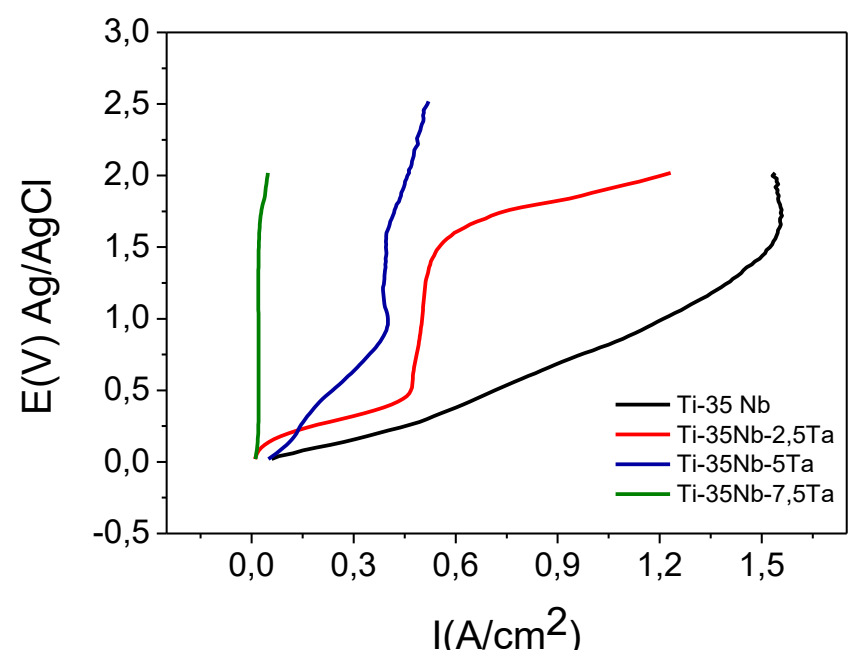


Figura 1. Curvas de polarização anódica das ligas sem anodizar

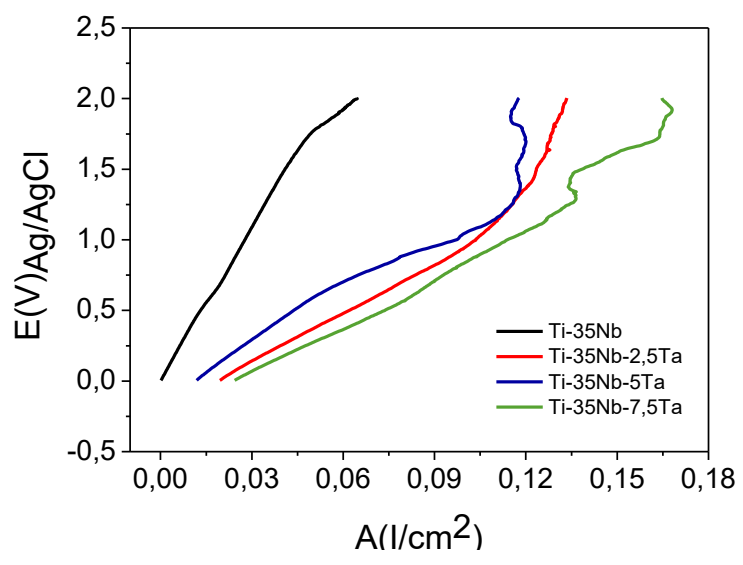

Figura 2. Curvas de polarização anódica das ligas anodizada

Para uma melhor visualização as curvas das ligas anodizadas e pós anodizadar foram plotados em pares conforme as figuras 3,4,5 e 6.E mais uma vez mostra que quando as ligas são anodizadas tempos uma densidade de corrente menor, logo uma maior resistência a corrosão. Para todas as ligas após o processo ouve uma melhora significativa nas densidades de corrente. Se tratando da liga sem a presença do elemento tântalo, Ti-35Nb surpreende trazendo uma grande diferença. Algo interessante ao se notar e que as ligas anodizadas possuem menos regiões de passivação que as curvas sem anodizar.Aplicar outras técnicas para maior conhecimento do filme formado e de grande interess.

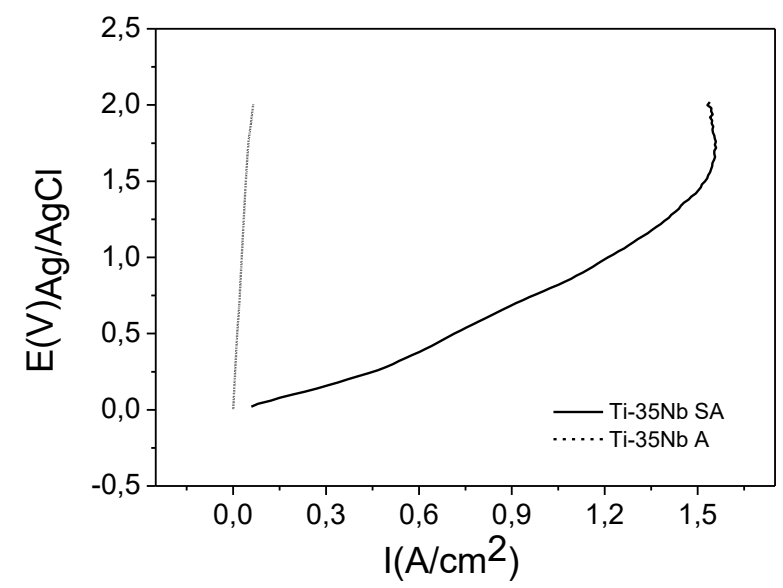


Figura 3: Ti-35Nb, sem anodizar e anodizada

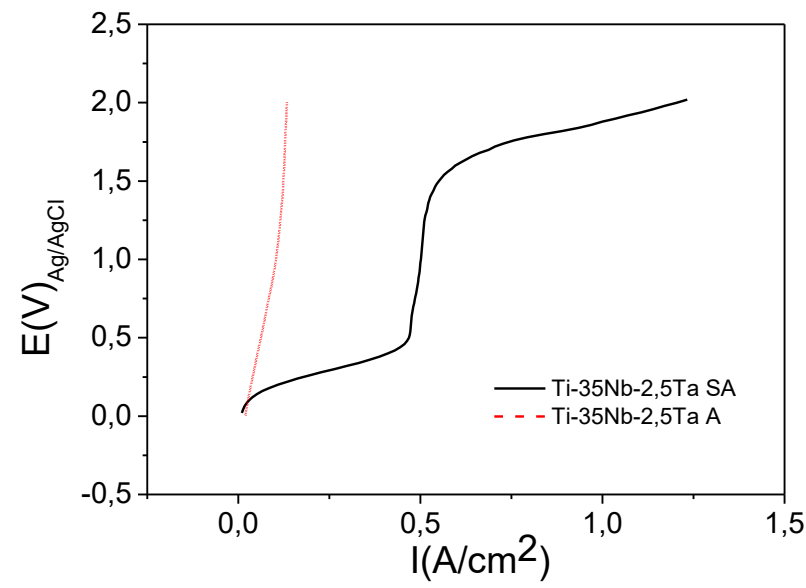

Figura 4: Ti-35Nb-2,5Ta, sem anodizar e anodizad

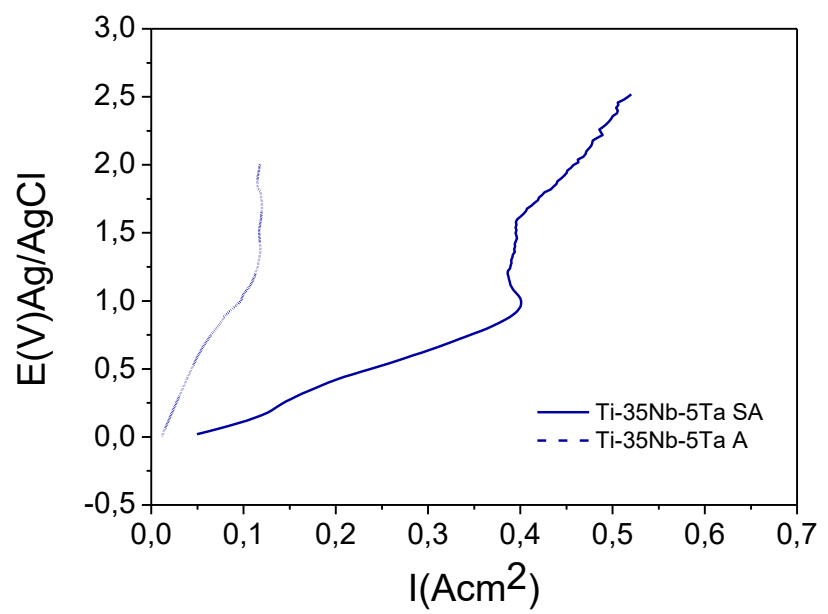

Figura 5: Ti-35Nb-5Ta, sem anodizar e anodizada

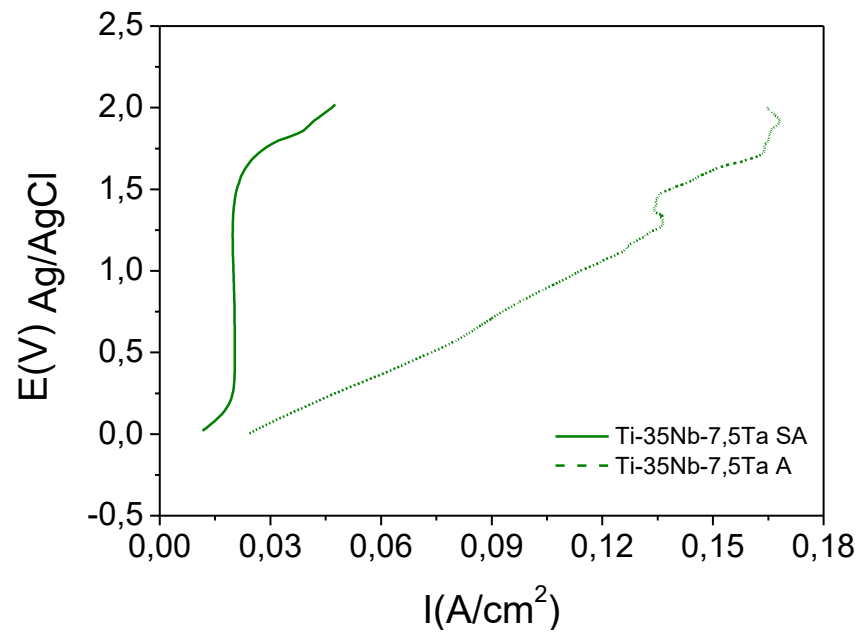

Figura 5: Ti-35Nb-7,5Ta sem anodizar e anodizada 


\section{CONCLUSÃO}

$>$ Todas as composições apresentam regiões de passivação e repassivação nas curvas de polarização anódica;

As ligas sem anodização apresentam mais regiões de passivação

$>\quad$ De acordo com os valores de densidade de corrente de corrosão todas as ligas mostram uma elevada resistência à corrosão no meio estudado;

$>\quad$ A resistência a corrosão tem um aumento significativo quando a anodização é aplicada pois as densidades de corrente diminuem quase 10 vezes.

$>\quad$ A liga de Ti-35Nb, apresenta uma resistência a corrosão menor que as ligas quando não esta anodizada e maior quando sofre a anodização.

> O oxido formado após as ligas passarem pelo processo de anodização apresenta uma grande resistência a corrosão. E mostra sofrer uma influencia do elemento Tântalo.

\section{AGRADECIMENTOS}

Ao CNPq pelo suporte financeiro.

\section{REFERÊNCIAS}

1

2 Frauchiger, V. M., et al. "Anodic plasma-chemical treatment of CP titanium surfaces for biomedical applications." Biomaterials 25.4 (2004): 593-606.

3 Grigal, I. P., et al. "Correlation between bioactivity and structural properties of titanium dioxide coatings grown by atomic layer deposition." Applied Surface Science 258.8 (2012): 3415-3419.

4 Kurtz, Steven M., et ai. "Futura demanda de pacientes jovens para substituição conjunta primária e de revisão: projeções nacionais de 2010 a 2030." Ortopedia Clínica e Pesquisa Relacionada® 467.10 (2009): 2606-2612.

5 Brammer, Karla S., Christine J. Frandsen e Sungho Jin. "Nanotubos TiO 2 para regeneração óssea". Tendências em biotecnologia 30,6 (2012): 315-322.

6 CHOE, H.-C.; JEONG, Y.-H.; BRANTLEY, W. A., 2010, "Phenomena of

7 nanotube nucleation and growth on new ternary titanium alloys", Journal of

8 Nanoscience and Nanotechnology, v. 10, n. 7, p. 4684-4689

9 Tavares A. M.G; Fernandes, B.S, Souza S.A; et al. The addition of Si to the Ti-35Nb alloy and its effect on the corrosion resistance, when applied to biomedical materials. Journal of Alloys and Compounds, 2014: 591: 91-99.

10 Geetha, M.,Singh,A.K.,Asokamanir. Et al.Ti based biomaterials, the utimate choice for orthopedic implants-A review.Progress in Materials science, 2009;54:397-425 